\section{(2) OPEN ACCESS}

\title{
Tissue bridges predict neuropathic pain emergence after spinal cord injury
}

\author{
Dario Pfyffer (1) , Kevin Vallotton, ${ }^{1}$ Armin Curt, ${ }^{1}$ Patrick Freund ${ }^{1,2,3,4}$
}

\begin{abstract}
${ }^{1}$ Spinal Cord Injury Center, University Hospital Balgrist, Zurich, Switzerland

${ }^{2}$ Department of Brain Repair and Rehabilitation, UCL Institute of Neurology, University College London, London, United Kingdom

${ }^{3}$ Wellcome Trust Centre for Neuroimaging, UCL Institute of Neurology, University College London, London, United Kingdom

${ }^{4}$ Department of Neurophysics, Max Planck Institute for Human Cognitive and Brain Sciences, Leipzig, Germany
\end{abstract}

\section{Correspondence to}

Professor Patrick Freund, Spinal Cord Injury Center, University Hospital Balgrist, Zurich 8008 , Switzerland; patrick.freund@ balgrist.ch

DP and KV contributed equally.

Received 3 March 2020

Revised 26 May 2020

Accepted 17 June 2020

Published Online First 11 August 2020

\section{Check for updates}

(C) Author(s) (or their employer(s)) 2020. Re-use permitted under CC BY. Published by BMJ.

To cite: Pfyffer D, Vallotton K, Curt A, et al. J Neurol Neurosurg Psychiatry 2020;91:1111-1117.

\begin{abstract}
Objective To assess associations between preserved spinal cord tissue quantified by the width of ventral and dorsal tissue bridges and neuropathic pain development after spinal cord injury.
\end{abstract}

Methods This retrospective longitudinal study includes 44 patients (35 men; mean (SD) age, 50.05 (18.88) years) with subacute (ie, 1 month) spinal cord injury (25 patients with neuropathic pain, 19 pain-free patients) and neuroimaging data who had a follow-up clinical assessment at 12 months. Widths of tissue bridges were calculated from midsagittal T2-weighted images and compared across groups. Regression analyses were used to identify relationships between these neuroimaging measures and previously assessed pain intensity and pin-prick score. Results Pin-prick score of the 25 patients with neuropathic pain increased from 1 to 12 months ( $\Delta$ mean $=10.08,95 \% \mathrm{Cl} 2.66$ to $17.50, p=0.010)$, while it stayed similar in pain-free patients $(\Delta$ mean $=2.74$, $95 \% \mathrm{Cl}-7.36$ to $12.84, \mathrm{p}=0.576)$. They also had larger ventral tissue bridges $(\Delta$ median $=0.80,95 \% \mathrm{Cl} 0.20$ to $1.71, p=0.008$ ) at 1 month when compared with pain-free patients. Conditional inference tree analysis revealed that ventral tissue bridges' width $(\leq 2.1$ or $>2.1 \mathrm{~mm})$ at 1 month is the strongest predictor for 12 months neuropathic pain intensity $(1.90 \pm 2.26$ and $3.83 \pm 1.19, p=0.042)$ and 12 months pin-prick score $(63.84 \pm 28.26$ and $92.67 \pm 19.43$, $p=0.025$ ).

Interpretation Larger width of ventral tissue bridges-a proxy for spinothalamic tract function - at 1 month postspinal cord injury is associated with the emergence and maintenance of neuropathic pain and increased pin-prick sensation. Spared ventral tissue bridges could serve as neuroimaging biomarkers of neuropathic pain and might be used for prediction and monitoring of pain outcomes and stratification of patients in interventional trials.

\section{INTRODUCTION}

Spinal cord injury (SCI) is a devastating and lifechanging event. In most cases, it produces immediate and permanent sensory, motor and autonomic dysfunction below the level of injury, resulting in a reduced quality of life. ${ }^{1}$ Along with the functional deficits, the majority of patients develops neuropathic pain (NP) at and/or below the level of injury. ${ }^{2}$ NP usually arises early after SCI, ${ }^{23}$ is often refractory to treatment $\mathrm{t}^{245}$ and normally persists over years ${ }^{3}$ with a potentially increasing intensity. ${ }^{6}$ Although the origin of NP is largely unknown, it is thought to arise at least partially in the spinal cord. ${ }^{78}$ Preserved tissue bridges adjacent to the intramedullary lesion cavity, which can be identified in all patients with incomplete SCI, ${ }^{9}$ are permissive for electrophysiological information flow and the size of which is predictive of functional recovery. ${ }^{9-11}$ Importantly, NP below the level of injury develops over time. ${ }^{12}$ This suggests that fractions of sensory pathways within preserved tissue bridges become active over time and may affect both ascending and descending modulatory systems. ${ }^{13-15}$ Moreover, hyperactive dorsal horn neurons of the spinothalamic tract have been identified as a possible pathobiological substrate. ${ }^{13}$ Interestingly, recovery of spinothalamic tract function (eg, pin-prick score) is enhanced in patients with SCI suffering from NP and its magnitude has been associated with pain intensity. ${ }^{16}$

Based on neuroimaging, the location and width of spared midsagittal tissue bridges have been shown to be critically involved in the recovery of sensorimotor function. ${ }^{9-11} 17$ For example, dorsal tissue bridges covering the dorsal columns are predictive of sensory evoked potentials and recovery of lighttouch function ${ }^{11}$ and may transmit pain signals evoked by normally non-painful stimuli (ie, allodynia). ${ }^{1819}$ Ventral tissue bridges have been linked to recovery of motor function ${ }^{11}$ and cover, next to the anterior corticospinal tract, ${ }^{20}$ also portions of the anterior spinothalamic tract. ${ }^{21}$ Thus, the question arises whether width of ventral and dorsal tissue bridges can predict the emergence and/or maintenance of NP after SCI.

This study aimed to explore the value of spared midsagittal tissue bridges (ventral and dorsal) derived from conventional T2-weighted (T2w) serial scans $^{9-11}$ in predicting the emergence and maintenance of SCI-related NP. Therefore, we investigated the relationships between the width of ventral and dorsal tissue bridges and pain intensity as well as its clinical characteristics.

\section{METHODS}

\section{Experimental design}

In this retrospective study, we included 44 patients with subacute SCI (28 tetraplegic and 16 paraplegic patients) who were admitted consecutively to the Balgrist University Hospital (Zurich, Switzerland) between May 1996 and January 2019. Of these 44 patients, all had a follow-up clinical assessment and 32 had a neuroimaging follow-up visit at 12 months post-SCI (figure 1).

We used 1 month and 12 months neuroimaging, neuropathic pain and clinical data from these patients to investigate group differences and clinicopathological relationships as well as outcome predictions by means of regression analysis and unbiased recursive partitioning (URP). Variables 


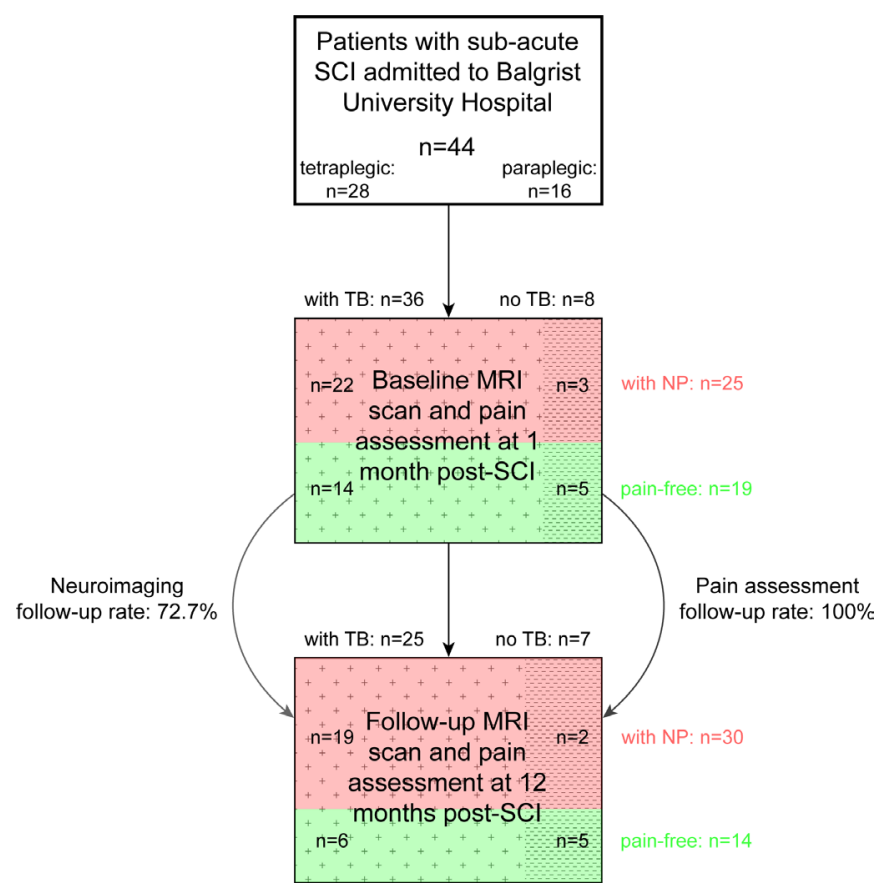

Figure 1 Study population with participant numbers, neuroimaging and pain characteristics as well as follow-up rate. Illustrative flow diagram showing the sample size of the study with subgroups according to their presence or absence of neuropathic pain (NP) and tissue bridges (TB). From 44 patients with subacute spinal cord injury (SCI), all had a follow-up pain assessment at 12 months, whereas only 32 underwent a follow-up MRI scan.

of interest included were lesion parameters as well as pain and clinical outcome measures at 1 month and 12 months follow-up.

We excluded patients with pre-existing neurological or mental disorders or brain lesions, as well as patients with MRI contraindications.

Tissue bridge data from a small subset of the study population was previously reported in a different context. ${ }^{9-11}$

\section{Clinical assessments}

All patients were clinically examined using a comprehensive clinical protocol including 1) the International Standards for Neurological Classification of Spinal Cord Injury protocol ${ }^{22}$ and 2) the European Multicenter Study about Spinal Cord Injury (EMSCI) pain questionnaire (V.4.2, http://www.emsci.org/). On the EMSCI questionnaire, patients rate various aspects of pain (eg, current pain intensity, mean and maximal pain intensity during the last week before the assessment, location and quality of pain, intensity of allodynia and paraesthesia). The pain intensity was quantified using an 11-point numeric rating scale with ' 0 ' indicating no pain and ' 10 ' indicating the worst imaginable pain. Data from the EMSCI pain questionnaire was available for 43 patients with SCI at 1 month (24 patients with NP, 19 painfree patients) and 42 patients at 12 months post-SCI ( 28 patients with NP, 14 pain-free patients). One patient and two patients without EMSCI pain questionnaire at 1 month and 12 months, respectively, reported to suffer from NP.

\section{Image acquisition}

All study participants underwent MRI on 1.5 or $3 \mathrm{~T}$ Philips (Philips Healthcare, Best, The Netherlands), Siemens (Siemens Healthcare, Erlangen, Germany) or GE (GE Medical Systems, Waukesha, Wisconsin, USA) scanners. A 32-channel receive spine coil integrated in the table was used with all scanners. The anatomical MRI protocol consisted of standard sagittal T1-weighted, sagittal T2w and axial T2w clinical scans obtained at the lesion level. Repetition time (TR), echo time (TE) and flip angle (FA) of the sagittal $\mathrm{T} 2 \mathrm{w}$ clinical sequences were used as follows: at $1.5 \mathrm{~T}(\mathrm{TR}=4138 \mathrm{~ms} ; \mathrm{TE}=109 \mathrm{~ms} ; \mathrm{FA}=149$ degrees $)$ and at $3 \mathrm{~T}(\mathrm{TR}=3938 \mathrm{~ms} ; \mathrm{TE}=97 \mathrm{~ms} ; \mathrm{FA}=153$ degrees $)$. The echo train length was 19 at $1.5 \mathrm{~T}$ and 17 at $3 \mathrm{~T}$. At 1.5 and $3 \mathrm{~T}$, the field of view9 was set to $330 \mathrm{~mm} \times 330 \mathrm{~mm}$ and 220 $\mathrm{mm} \times 220 \mathrm{~mm}$, respectively. To reduce metal artefacts, readout bandwidths were increased at $1.5 \mathrm{~T}(415 \mathrm{~Hz} /$ pixel $)$ and at $3 \mathrm{~T}$ $(751 \mathrm{~Hz} /$ pixel). The spatial resolution of sagittal $\mathrm{T} 2 \mathrm{w}$ images was $0.55 \mathrm{~mm} \times 0.55 \mathrm{~mm} \times 2.75 \mathrm{~mm}$ at $1.5 \mathrm{~T}$ and $0.57 \mathrm{~mm} \times 0.57$ $\mathrm{mm} \times 2.75 \mathrm{~mm}$ at $3 \mathrm{~T}$. The midsagittal slices of sagittal T2 $\mathrm{w}$ scans were the ones used for lesion segmentation analysis. ${ }^{9-11}$

\section{Image analysis}

Together with oedema and haemorrhage, intramedullary damage manifests as changes of signal intensity on T2w scans. ${ }^{23}$ Crucially, hyperintense signal changes in the subacute phase reliably reflect neural damage within the spinal $\operatorname{cord}^{9}{ }^{10}$ rather than oedema and haemorrhage which is harder to differentiate in the acute phase after SCI. Patients' neuroimaging data were only included if T2w scans showed a clearly visible lesion (ie, hyperintense signal)_if present-on the midsagittal slice whereas scans with insufficient image quality or lesion visibility due to metal artefacts were excluded. ${ }^{9-11}$ Neuroimaging data with appropriate image quality and lesion visibility was available for 44 patients with subacute SCI of which 32 had a follow-up scan at 12 months post-SCI.

Lesion segmentation was performed manually and blinded to patient identity and scan time point by rater DP. We used the Jim software (V.7.0, Xinapse Systems, Aldwincle, UK) to delineate the lesion on the midsagittal slice from sagittal T2w scans. This enabled us to assess the lesion area, its rostro-caudal lesion length, anterior-posterior lesion width and ventral and dorsal tissue bridges, the sum of both reflecting the total width of tissue bridges (ie, hypointense regions between the relatively hyperintense adjacent cystic cavity within the spinal cord and the cerebrospinal fluid)..$^{9} 10$

\section{Statistical analysis}

Statistical analysis was performed using Stata software (V.4.2; StataCorp, College Station, Texas, USA). We used unpaired twotailed t-tests to compare patients with SCI with NP and pain-free patients with SCI regarding their age at 1 month scanning time point and the time interval between injury and 1 month scan. Paired two-tailed t-tests were used for comparison of midsagittal tissue bridges (ie, total, ventral and dorsal), pin-prick score and mean pain intensity at 1 month and 12 months post-SCI.

We applied Mann-Whitney U tests for pairwise comparisons of patient groups regarding their pin-prick score and width of midsagittal tissue bridges (ie, ventral, dorsal and total) at 1 month and 12 months post-SCI as well as rate of change in pinprick score from 1 month to 12 months.

We used multivariable linear regression analysis to investigate associations between imaging measures (ie, width of ventral midsagittal tissue bridges) and mean pain intensity at 1 month. One month quantitative lesion measures were also used with the same model to predict mean NP intensity and recovery of pain sensation (ie, pin-prick score) at 12 months follow-up. Age and sex were included as covariates of no interest in the regression models to adjust for linear age and sex dependency. Models including ventral tissue bridges were also corrected for dorsal 
tissue bridges. Prediction models were furthermore adjusted for 1 month clinical scores (eg, pin-prick score at 1 month). Potential confounders were only retained if the covariates were significant or if they had a substantial effect on the correlation coefficient of interest. Results were regarded as significant when the $\mathrm{p}$ values were $\leq 0.05$ and CIs were set to $95 \%$.

We further used a widely used ${ }^{24-28}$ unbiased recursive partitioning technique called conditional inference tree (URPCTREE) ${ }^{29}$ implemented in the 'party' package within $\mathrm{R}^{30}$ (V.3.4.3). URP-CTREE performs a prospective prediction-based stratification of a patient population with regard to predefined outcome variables. URP-CTREE is a tree-structured regression model for independence tests between sets of predictors (eg, early imaging parameters) and specified future clinical end points (eg, pain sensation measures). We used 1 month ventral and dorsal midsagittal tissue bridges' widths as predictors for mean neuropahtic pain intensity and pin-prick score at 1 month and 12 months. The URP-CTREE algorithm dichotomously separates an initial heterogeneous patient population into more homogeneous and well-defined pairs of subgroups (ie, nodes) with respect to the future clinical outcomes specified. Subgroups that will be further separated are referred to as inner nodes. Separation goes on as long as one of the predictor variables is found to be associated with the predefined clinical end point and to split the group into two disjoint subgroups with a $\mathrm{p}$ value $\leq 0.05$. The algorithm is designed in a way that every separation is based on the singular most significant predictor with the aim to maximise the difference between the newly formed subgroups. Splitting groups is continued as long as there is any significant predictor value.

\section{Data availability}

Anonymised data of this study are available on request from the corresponding author.

\section{RESULTS}

Demographics and clinical characteristics

Forty-four patients with SCI $(\mathrm{n}=35$ men $(79.5 \%))$ with a mean $( \pm S D)$ age of $50.05 \pm 18.88$ years were included. Patients with NP $(50.40 \pm 17.38$ years, $\mathrm{n}=25)$ and pain-free patients $(49.58 \pm 21.17$ years, $\mathrm{n}=19)$ did not differ in their age at 1 month $(\Delta$ mean $=-0.82,95 \% \mathrm{CI}-12.55$ to $10.91, \mathrm{p}=0.888)$. The patients' time interval between injury and 1 month scan (ie, subacute stage) was $32.95 \pm 16.85$ days. There was no difference in time since injury $(\Delta$ mean $=4.80,95 \%$ CI -5.56 to 15.17 , $\mathrm{p}=0.355)$ between patients with NP $(30.88 \pm 17.55$ days, $\mathrm{n}=25)$ and pain-free patients $(35.68 \pm 15.93$ days, $\mathrm{n}=19)$.

Over time, NP developed in six initially pain-free patients whereas one patient with NP at baseline reported to be NP-free at follow-up. Patients with NP at 1 month (56.8\%) and 12 months follow-up (68.2\%) reported a similar mean NP intensity of $3.67 \pm 1.61$ and $3.68 \pm 1.61(\Delta$ mean $=0.01,95 \% \mathrm{CI}-0.89$ to $0.91, \mathrm{p}=0.979$ ), respectively. Pin-prick score of patients with SCI with NP was $70.08 \pm 28.40$ at 1 month and increased to $80.16 \pm 27.88$ at 12 months post-SCI $(\Delta$ mean $=10.08,95 \%$ CI 2.66 to $17.50, \mathrm{p}=0.010, \mathrm{n}=25$ ). In pain-free patients, 1 month pin-prick score was $57.84 \pm 25.02$ and did not significantly change over time $(60.58 \pm 27.25$ at follow-up $(\Delta$ mean $=2.74$, $95 \%$ CI -7.36 to $12.84, \mathrm{p}=0.576, \mathrm{n}=19))$. Between both patient groups, there was no difference in pin-prick score at 1 month ( $\Delta$ median $=15.00,95 \% \mathrm{CI}-7.00$ to $29.00, \mathrm{p}=0.184$ ) and the rate of change in pin-prick score from 1 month to follow-up at 12 months did not differ $(\Delta$ median $=3.00,95 \%$ CI -3.00 to $12.00, \mathrm{p}=0.286$ ).

The role of midsagittal tissue bridges in NP

Of 44 patients, 8 (ie, American Spinal Injury Association Impairment Scale (AIS) A patients) had no midsagittal tissue bridges (18.2\%) while the remaining 36 (ie, 1 AIS A and 35 AIS B-D patients) did have midsagittal tissue bridges (81.8\%) with an average width of $2.21 \pm 1.65 \mathrm{~mm}$. Thirty-one of $44(70.5 \%)$ patients had ventral tissue bridges with an average width of $1.17 \pm 1.18 \mathrm{~mm}$ and 29 of $44(65.9 \%)$ had dorsal tissue bridges with an average width of $1.03 \pm 1.20 \mathrm{~mm}$. Twenty-four patients had both ventral and dorsal tissue bridges while seven and five patients had only ventral or dorsal bridges, respectively.

At 1 month, width of total midsagittal tissue bridges was larger $(\Delta$ median $=1.15,95 \%$ CI 0.00 to $2.12, p=0.034$, figure $2 \mathrm{~A})$ in patients with SCI with NP $(2.67 \pm 1.71 \mathrm{~mm}$, $\mathrm{n}=25)$ when compared with pain-free patients $(1.60 \pm 1.38 \mathrm{~mm}$, $\mathrm{n}=19)$. At 1 month, ventral tissue bridges of patients with NP $(1.60 \pm 1.25 \mathrm{~mm}, \mathrm{n}=25)$ were larger $(\Delta$ median $=0.80,95 \% \mathrm{CI}$ 0.20 to $1.71, \mathrm{p}=0.008)$ in comparison to pain-free patients $(0.61 \pm 0.81 \mathrm{~mm}, \mathrm{n}=19)$. Dorsal tissue bridges at 1 month were similar $(\Delta$ median $=0.00,95 \% \mathrm{CI}-0.69$ to $0.50, \mathrm{p}=0.790)$ in patients with NP $(1.07 \pm 1.33 \mathrm{~mm}, \mathrm{n}=25)$ and pain-free patients $(0.99 \pm 1.03 \mathrm{~mm}, \mathrm{n}=19)$.

Of 25 patients with SCI with NP and 19 pain-free patients, 17 and 15 had imaging follow-up data, respectively (table 1). In the 17 patients with NP, width of total tissue bridges was similar at 1 month and 12 months post-SCI $(\Delta$ mean $=0.29 \mathrm{~mm}, 95 \% \mathrm{CI}$ -0.12 to $0.69 \mathrm{~mm}, \mathrm{p}=0.153$, figure $2 \mathrm{~B}$ ). Within the pain-free group, width of total tissue bridges was also comparable between 1 month and 12 months follow-up $(\Delta$ mean $=-0.48 \mathrm{~mm}, 95 \% \mathrm{CI}$ -1.02 to $0.07 \mathrm{~mm}, \mathrm{p}=0.081$ ). Width of ventral tissue bridges was comparable at 1 month and 12 months in patients with NP $(\Delta$ mean $=-0.04 \mathrm{~mm}, 95 \% \mathrm{CI}-0.39$ to $0.31 \mathrm{~mm}, \mathrm{p}=0.816$, figure $2 \mathrm{C})$ and pain-free patients $(\Delta$ mean $=-0.33 \mathrm{~mm}, 95 \% \mathrm{CI}$ -0.73 to $0.07 \mathrm{~mm}, \mathrm{p}=0.097$ ). Similarly, the rate of change in width of dorsal tissue bridges over time was similar for patients with NP $(\Delta$ mean $=0.32 \mathrm{~mm}, 95 \%$ CI 0.00 to $0.65 \mathrm{~mm}, \mathrm{p}=0.053$, figure $2 \mathrm{D})$ and pain-free patients $(\Delta$ mean $=-0.15 \mathrm{~mm}, 95 \% \mathrm{CI}$ -0.43 to $0.13 \mathrm{~mm}, \mathrm{p}=0.280$ ).

\section{Association of tissue bridges and clinical pain measures at 1 month}

Larger width of ventral tissue bridges at 1 month was associated with a higher mean pain intensity at 1 month (coefficient $=0.23$, $95 \%$ CI 0.07 to $0.39, \mathrm{p}=0.005, \mathrm{R}^{2}=0.179, \mathrm{n}=43$, figure $3 \mathrm{~A}$ ), adjusted for dorsal tissue bridges and age.

URP-CTREE analysis led to a partition of the entire cohort $(\mathrm{p}=0.013, \mathrm{n}=43$, figure $3 \mathrm{~B})$ into two terminal nodes with regard to the patients' mean pain intensities (ranging from 0 to 10) at 1 month, according to the 1 month width of ventral tissue bridges being $\leq 2.1 \mathrm{~mm}(\mathrm{n}=31$, node 2$)$ or $>2.1 \mathrm{~mm}(\mathrm{n}=12$, node 3 ). The two subgroups presented 1 month mean NP intensities of $1.45 \pm 2.05$ and $3.58 \pm 1.83$, respectively.

Association of tissue bridges and clinical pain measures at 12 months

Larger ventral tissue bridges at 1 month were associated with a higher mean NP intensity (coefficient $=0.20,95 \%$ CI 0.03 to $0.36, \mathrm{p}=0.022, \mathrm{R}^{2}=0.131, \mathrm{n}=42$ ) and higher pin-prick score at 12 months (coefficient $=0.03,95 \%$ CI 0.01 to $0.04, \mathrm{p}<0.001$, $\mathrm{R}^{2}=0.355, \mathrm{n}=44$ ), independent of dorsal tissue bridges, age and 1 month clinical score. When looking at subgroups separately, 

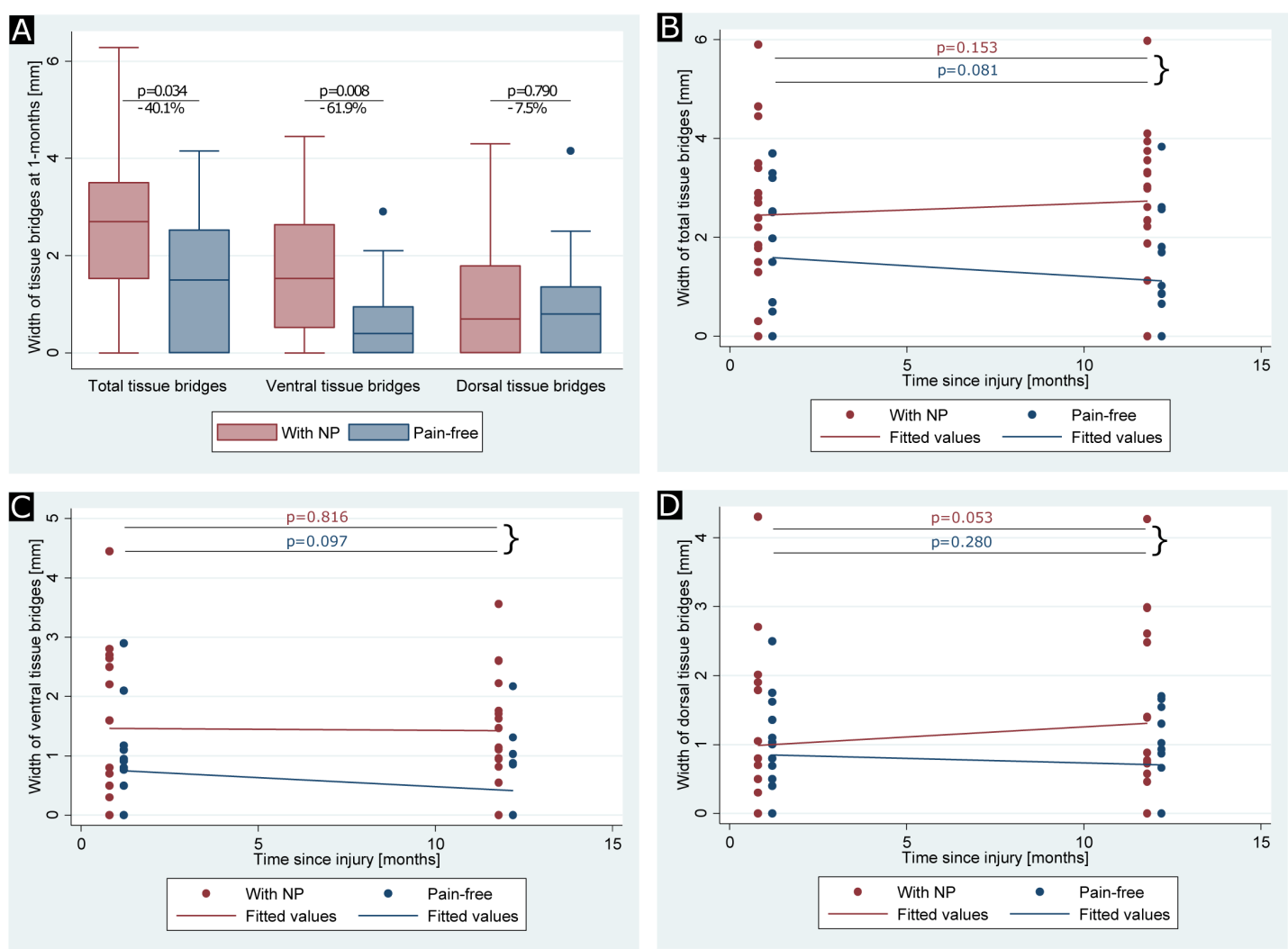

Figure 2 Between and within group comparison of tissue bridges at 1 month and 12 months. (A) Group differences of total, ventral and dorsal tissue bridges are shown for patients with spinal cord injury with neuropathic pain (NP) (indicated in red) and pain-free patients (indicated in blue). (B-D) Spatiotemporal evolutions of (B) ventral, (C) dorsal and (D) total tissue bridges from 1 month to 12 months are shown for patients with spinal cord injury with NP (indicated in red) and pain-free patients (indicated in blue) separately. Uncorrected p values are reported for significant differences.

patients with NP (coefficient $=0.03$, 95\% CI 0.01 to 0.04 , $\mathrm{p}=0.007, \mathrm{R}^{2}=0.385, \mathrm{n}=30$, figure $4 \mathrm{~A}$ ), but not pain-free patients (coefficient $=-0.01,95 \%$ CI -0.03 to $0.02, \mathrm{p}=0.525$, $\mathrm{R}^{2}=-0.386, \mathrm{n}=14$ ) showed a positive relationship between width of ventral tissue bridges at 1 month and pin-prick score at 12 months.

URP-CTREE analysis separated the entire cohort $(\mathrm{p}=0.042$, $\mathrm{n}=42$ ) into two terminal nodes with regard to the patients' mean pain intensities at 12 months, according to the 1 month width of ventral tissue bridges being $\leq 2.1 \mathrm{~mm}(\mathrm{n}=30$, node 2$)$ or $>2.1 \mathrm{~mm}$ $(\mathrm{n}=12$, node 3$)$. The two subgroups presented 12 months mean pain intensities of $1.90 \pm 2.26$ and $3.83 \pm 1.19$, respectively. The URP-CTREE algorithm also identified 1 month ventral tissue bridges as a predictor for 12 months pin-prick score, its width separating the initial group $(\mathrm{p}=0.025, \mathrm{n}=44$, figure $4 \mathrm{~B})$ into two nodes with $\leq 2.1 \mathrm{~mm}(\mathrm{n}=32$, node 2$)$ and $>2.1 \mathrm{~mm}(\mathrm{n}=12$, node 5 ) in ventral tissue bridges' width. The two subgroups presented 12 months pin-prick scores of $63.84 \pm 28.26$ and $92.67 \pm 19.43$, respectively. In a second step, the algorithm identified width of dorsal tissue bridges as a second predictor variable $(p=0.030)$ for the subgroup showing a width of ventral tissue bridges $\leq 2.1 \mathrm{~mm}$ and separated it into two more nodes with $\leq 1.62 \mathrm{~mm}(\mathrm{n}=24$, node 3$)$ and $>1.62 \mathrm{~mm}(\mathrm{n}=8$, node 4$)$ in dorsal tissue bridges' width. The two subgroups presented 12 months pin-prick scores of $56.58 \pm 27.18$ and $85.63 \pm 19.66$, respectively.

\section{DISCUSSION}

This study demonstrates the potential involvement of spared ventral tissue bridges in the development and maintenance of NP following SCI. In particular, patients with NP had larger total and ventral tissue bridges when compared with pain-free patients, their width at 1 month being a predictor for a higher NP intensity and higher pin-prick score at 12 months post-SCI.

As expected, ${ }^{2} 31$ about two-third (68.2\%) of the patients with SCI enrolled in this study developed NP of which more than half had already NP at the subacute stage (56.8\%). Hari et al ${ }^{16}$ reported that enhanced recovery of spinothalamic tract function (ie, pin-prick) is associated with NP after SCI, and Hatem et al ${ }^{32}$ showed that there were less spinothalamic tract impairments in patients with syringomyelia with both spontaneous and evoked pain when compared with patients with spontaneous pain only. In our study, recovery of pin-prick score (ie, clinical measure for

Table 1 Dynamic change of width of total, ventral and dorsal tissue bridges

\begin{tabular}{|c|c|c|c|c|}
\hline \multirow[b]{2}{*}{ Width of tissue bridges } & \multicolumn{2}{|c|}{ Patients with SCI with NP $(n=17)$} & \multicolumn{2}{|c|}{ Pain-free patients with $\mathrm{SCl}(\mathrm{n}=15)$} \\
\hline & 1 month & 12 months & 1 month & 12 months \\
\hline Total, mean (SD), mm & $2.45(1.64)$ & $2.73(1.48)$ & $1.60(1.33)$ & $1.12(1.17)$ \\
\hline Ventral, mean (SD), mm & $1.46(1.29)$ & $1.42(0.94)$ & $0.75(0.86)$ & $0.42(0.68)$ \\
\hline Dorsal, mean (SD), mm & $0.99(1.20)$ & $1.31(1.29)$ & $0.85(0.74)$ & $0.70(0.66)$ \\
\hline
\end{tabular}

$\mathrm{NP}$, neuropathic pain; $\mathrm{SCl}$, spinal cord injury. 

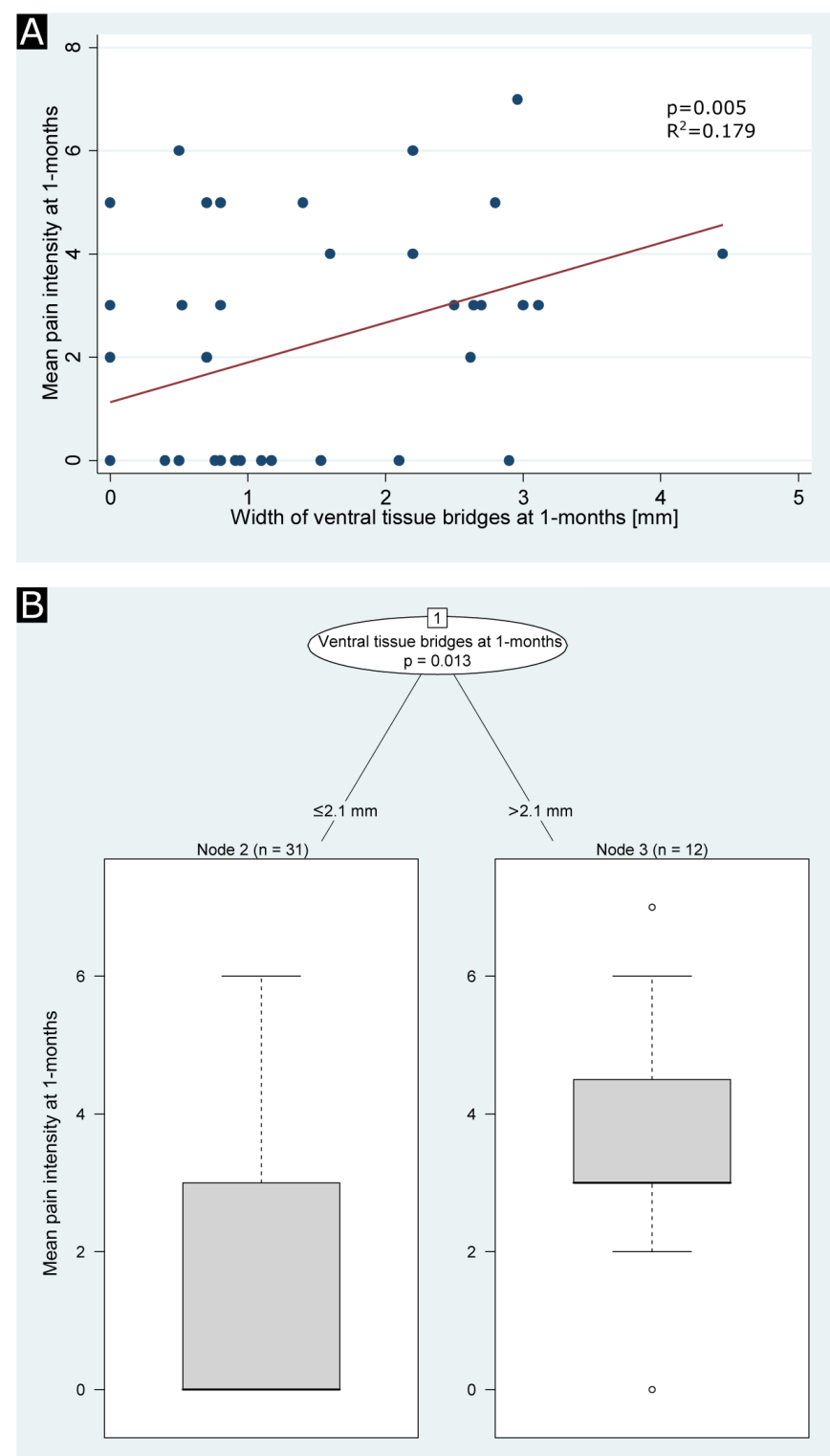

Figure 3 Regression analyses between width of tissue bridges and mean pain intensity at 1 month. (A) Regression graph showing the association of mean pain intensity at 1 month with width of ventral tissue bridges at 1 month for all patients with spinal cord injury. The red line indicates the linear fit. (B) Unbiased recursive partitioning conditional inference tree for the clinical end point mean pain intensity at 1 month of patients with spinal cord injury with available pain intensity data at 1 month $(n=43)$. Ventral and dorsal tissue bridges' widths at 1 month were used as predictors in the model. The algorithm led to a partition of the initial patient population (node 1) into two terminal nodes (nodes 2 and 3 ) representing the more homogeneous subgroups. Box plots at the bottom show sizes of the subgroups, indicated above each terminal node, with their corresponding distributions (including two-sided error bars) of the clinical end point. Uncorrected $p$ values are reported in $(A)$ and multiple testing-corrected (ie, Bonferroni-corrected) $p$ values are reported in (B) for significant differences.

pain sensation) paralleled the emergence of NP and its intensity while no substantial change was observed in the pain-free group. This supports the notion that recovery of spinothalamic function is involved in the emergence and/or maintenance of NP. ${ }^{81632-34}$

Ventral tissue bridge measures are likely to cover the anterior spinothalamic tract. ${ }^{21}$ Interestingly, patients with NP had greater
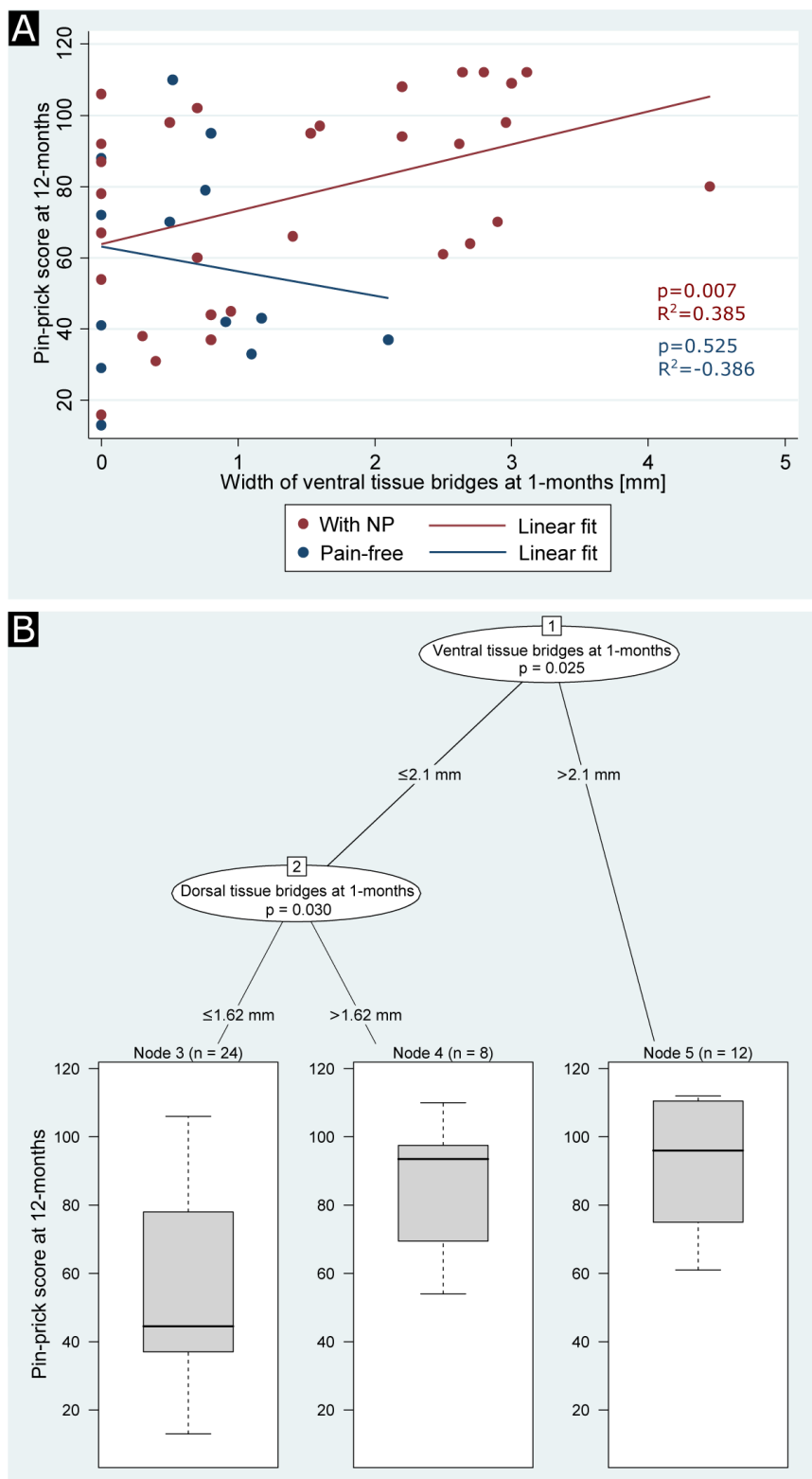

Figure 4 Regression analyses between width of tissue bridges at 1 month and pin-prick score at 12 months. (A) Regression graph showing the association of pin-prick score at 12 months with width of ventral tissue bridges at 1 month for patients with spinal cord injury with neuropathic pain (NP) (indicated in red) and pain-free patients (indicated in blue). The red and blue lines indicate the corresponding linear fits. (B) Unbiased recursive partitioning conditional inference tree for the clinical end point pin-prick score at 12 months of all patients with spinal cord injury ( $n=44)$. Ventral and dorsal tissue bridges' widths at 1 month were used as predictors in the model. The algorithm led to a partition of the initial patient population (node 1) into one more inner node (node 2) and finally into three terminal nodes (nodes 3,4 and 5) representing the more homogeneous subgroups. Box plots at the bottom show sizes of the subgroups, indicated above each terminal node, with their corresponding distributions (including two-sided error bars) of the clinical end point. Uncorrected $p$ values are reported in $(A)$ and multiple testing-corrected (ie, Bonferroni-corrected) $p$ values are reported in (B) for significant differences.

width of ventral tissue bridges at baseline and follow-up, while the width of dorsal tissue bridges was similar between groups at both time points. Crucially, larger ventral tissue bridges at 1 month are associated with higher mean pain intensities at 1 
month and 12 months, independent of dorsal tissue bridges. By means of URP-CTREE analysis, we further identified ventral tissue bridges at 1 month as predictors of recovery of pin-prick scores at 12 months with a cut-off value of $2.1 \mathrm{~mm}$. Next to the implication of ventral tissue bridges, dorsal tissue bridges might also be involved in the generation of NP. ${ }^{1819}$ To examine pin-prick sensation (ie, clinical pain measure), the patient needs to distinguish a sharp and a dull end of a pin when blindfolded. ${ }^{22}$ Thus, next to spinothalamic tract function, the medial lemiscal system running through the dorsal columns (ie, dorsal tissue bridges) is also involved as it conveys the information for tactile discrimination. ${ }^{35}$ Thus, dorsal tissue bridges likely involve second-order neurons of the spinothalamic tract within the substantia gelatinosa of the spinal cord dorsal horn. ${ }^{36}$ Sparing of these neurons could be of high importance for maintenance or recovery of clinical pain sensation (ie, pin-prick score) at dermatomes corresponding to the lesioned spinal cord levels. Finnerup et al ${ }^{37}$ reported that patients with SCI with NP, when compared with pain-free patients with SCI, showed more sensory hypersensitivity in lesion level-related dermatomes, suggesting a key role of sensory neuronal hyperexcitability in NP following SCI. ${ }^{38-40}$

This study has limitations. First of all, this study is a retrospective monocentric study with specified inclusion criteria. This likely represents a bias source as the study design resulted in a homogeneous data set, but might not reflect the general SCI population. Future studies would therefore benefit from a prospective multicentric design including larger patient numbers. Furthermore, women and men were not equally distributed in our patient cohort. However, this is also not the case for the general SCI population. ${ }^{41}$ Quantification of tissue bridges was only performed on midsagittal but not parasagittal slices. Further quantification of lateral tissue bridges on T2w axial slices was also not possible due to a low spatial resolution. This limits information about specific spared tracts and potential correlations with their corresponding functions. These points should be addressed in future studies to further explore the already promising method of tissue bridges characterisation $^{9-1117}$ with its reliable quantification already at early stages after SCI and even in the presence of metal artefacts near the lesion. ${ }^{10}$ The EMSCI pain questionnaire rather crudely assesses NP characteristics. Future studies would benefit from using more detailed pain assessments including drawings of the body with the extent, quality and intensity of the perceived pain at different body parts. ${ }^{42}$ Finally, even though our sample size was rather large, including more patients in the URP-CTREE analysis would increase the chances of reliably predicting future clinical end points and stratifying a heterogeneous patient cohort into homogeneous subgroups via multilevel inference trees and corresponding predictor variables.

This study identifies spared tissue bridges as quantifiable neuroimaging biomarkers assessed by conventional MRI predicting the emergence and maintenance of NP. Specifically, we identified 1 month width of ventral tissue bridges as the strongest predictor of NP intensity and pin-prick score at 12 months post-SCI. Thus, ventral tissue bridges represent MRI metrics, which could be readily implemented in the daily clinical routine to serve as promising, reliable and time-saving neuroimaging biomarkers for the monitoring of NP and for stratification of patient subgroups in interventional clinical trials.

Correction notice This paper has been corrected since it appeared Online First. License has been corrected to CC-BY.

Acknowledgements The authors would like to thank the whole European Multicenter study about Spinal Cord Injury (EMSCI) Study Group and in particular
René Koller for the assistance with the EMSCI database. The authors would like to thank the staff of the Department of Neurology and Radiology at the Balgrist University Hospital for their great help. The authors would also like to thank all study participants for their time spent and contribution and DP, Balgrist University Hospital, ETH and University of Zurich and Kevin Vallotton, MD, Balgrist University Hospital, University of Zurich for conducting statistical analysis.

Contributors DP: study concept and design; acquisition, analysis and interpretation of data; writing the manuscript. KV: study concept and design; acquisition, analysis and interpretation of data; writing the manuscript. AC: critical revision of manuscript for intellectual content. PF: study concept and design; study supervision; critical revision of manuscript for intellectual content.

Funding This research was supported by Wings for Life, Austria (WFL-CH-007/14), the International Foundation for Research in Paraplegia (IRP-P158), the EU project (Horizon2020 'NISCl' grant agreement no. 681094) and the framework of ERA-NET NEURON (BMBF, 01EW1711A and B). PF is funded by an SNF Eccellenza Professorial Fellowship grant (PCEFP3_181362/1). Open access of this publication is supported by the Wellcome Trust (091593/Z/10/Z).

\section{Competing interests None declared.}

\section{Patient consent for publication Not required.}

Ethics approval The local ethics committee approved the study protocol (EK2010-0271), which was conducted in accordance with the Declaration of Helsinki and all patients gave informed, written consent prior to study enrolment.

Provenance and peer review Not commissioned; externally peer reviewed.

Data availability statement Data are available on reasonable request. Anonymised data of this study are available on request from the corresponding author.

Open access This is an open access article distributed in accordance with the Creative Commons Attribution 4.0 Unported (CC BY 4.0) license, which permits others to copy, redistribute, remix, transform and build upon this work for any purpose, provided the original work is properly cited, a link to the licence is given, and indication of whether changes were made. See: https://creativecommons.org/ licenses/by/4.0/.

\section{ORCID iD}

Dario Pfyffer http://orcid.org/0000-0002-2406-9251

\section{REFERENCES}

1 Ahuja CS, Wilson JR, Nori S, et al. Traumatic spinal cord injury. Nat Rev Dis Primers 2017;3:17018.

2 Burke D, Fullen BM, Stokes D, et al. Neuropathic pain prevalence following spinal cord injury: a systematic review and meta-analysis. Eur J Pain 2017;21:29-44.

3 Störmer S, Gerner HJ, Grüninger W, et al. Chronic pain/dysaesthesiae in spinal cord injury patients: results of a multicentre study. Spinal Cord 1997;35:446-55.

4 Mehta S, Orenczuk K, McIntyre A, et al. Neuropathic pain post spinal cord injury Part 1: systematic review of physical and behavioral treatment. Top Spinal Cord Inj Rehabil 2013;19:61-77.

5 Gruener H, Zeilig G, Gaidukov E, et al. Biomarkers for predicting central neuropathic pain occurrence and severity after spinal cord injury: results of a long-term longitudinal study. Pain 2020;161:545-56.

6 Grabher P, Callaghan MF, Ashburner J, et al. Tracking sensory system atrophy and outcome prediction in spinal cord injury. Ann Neurol 2015;78:751-61.

7 Tsuda M. Microglia in the spinal cord and neuropathic pain. J Diabetes Investig 2016;7:17-26.

8 Gwak YS, Hulsebosch CE, Leem JW. Neuronal-Glial interactions maintain chronic neuropathic pain after spinal cord injury. Neural Plast 2017;2017:2480689.

9 Huber E, Lachappelle P, Sutter R, et al. Are midsagittal tissue bridges predictive of outcome after cervical spinal cord injury? Ann Neurol 2017;81:740-8.

10 Pfyffer $D$, Huber $E$, Sutter $R$, et al. Tissue bridges predict recovery after traumatic and ischemic thoracic spinal cord injury. Neurology 2019;93:e1550-60.

11 Vallotton $\mathrm{K}$, Huber E, Sutter R, et al. Width and neurophysiologic properties of tissue bridges predict recovery after cervical injury. Neurology 2019;92:e2793-802.

12 Finnerup NB, Norrbrink C, Trok K, et al. Phenotypes and predictors of pain following traumatic spinal cord injury: a prospective study. J Pain 2014;15:40-8.

13 Wasner $\mathrm{G}$, Lee BB, Engel $\mathrm{S}$, et al. Residual spinothalamic tract pathways predict development of central pain after spinal cord injury. Brain 2008;131:2387-400.

14 Wrigley PJ, Siddall PJ, Gustin SM. New evidence for preserved somatosensory pathways in complete spinal cord injury: a fMRI study. Hum Brain Mapp 2018;39:588-98.

15 Wrigley PJ, Press SR, Gustin SM, et al. Neuropathic pain and primary somatosensory cortex reorganization following spinal cord injury. Pain 2009;141:52-9.

16 Hari AR, Wydenkeller S, Dokladal P, et al. Enhanced recovery of human spinothalamic function is associated with central neuropathic pain after SCl. Exp Neurol 2009:216:428-30 
17 O'Dell DR, Weber KA, Berliner JC, et al. Midsagittal tissue bridges are associated with walking ability in incomplete spinal cord injury: a magnetic resonance imaging case series. J Spinal Cord Med 2020;43:268-71.

18 Kim J, Back SK, Yoon YW, et al. Dorsal column lesion reduces mechanical allodynia in the induction, but not the maintenance, phase in spinal hemisected rats. Neurosci Lett 2005;379:218-22.

19 Sun $\mathrm{H}$, Ren K, Zhong CM, et al. Nerve injury-induced tactile allodynia is mediated via ascending spinal dorsal column projections. Pain 2001:90:105-11.

20 Welniarz Q, Dusart I, Roze E. The corticospinal tract: evolution, development, and human disorders. Dev Neurobiol 2017;77:810-29.

21 Saliani A, Perraud B, Duval T, et al. Axon and myelin morphology in animal and human spinal cord. Front Neuroanat 2017;11:129.

22 Kirshblum SC, Burns SP, Biering-Sorensen F, et al. International standards for neurological classification of spinal cord injury (revised 2011). J Spinal Cord Med 2011:34:535-46.

23 Goldberg AL, Daffner RH, Schapiro RL. Imaging of acute spinal trauma: an evolving multi-modality approach. Clin Imaging 1990;14:11-16.

24 De Roock W, Claes B, Bernasconi D, et al. Effects of KRAS, BRAF, NRAS, and PIK3CA mutations on the efficacy of cetuximab plus chemotherapy in chemotherapy-refractory metastatic colorectal cancer: a retrospective Consortium analysis. Lancet Oncol 2010;11:753-62.

25 Brown AW, Malec JF, McClelland RL, et al. Clinical elements that predict outcome after traumatic brain injury: a prospective multicenter recursive partitioning (decision-tree) analysis. J Neurotrauma 2005:22:1040-51.

26 Tanadini LG, Hothorn T, Jones LAT, et al. Toward inclusive trial protocols in heterogeneous neurological disorders: Prediction-Based stratification of participants with incomplete cervical spinal cord injury. Neurorehabil Neural Repair 2015:29:867-77.

27 Tanadini LG, Steeves JD, Hothorn T, et al. Identifying homogeneous subgroups in neurological disorders: unbiased recursive partitioning in cervical complete spinal cord injury. Neurorehabil Neural Repair 2014;28:507-15.

28 Velstra I-M, Bolliger M, Tanadini LG, et al. Prediction and stratification of upper limb function and self-care in acute cervical spinal cord injury with the graded redefined assessment of strength, sensibility, and prehension (GRASSP). Neurorehabil Neural Repair 2014:28:632-42.
29 Hothorn T, Hornik K, Zeileis A. Unbiased recursive partitioning: a conditional inference framework. J Comput Graph Stat 2006;15:651-74.

30 R Core Team. R: A Language and Environment for Statistical Computing [program]. Vienna, Austria: R Foundation for Statistical Computing, 2016.

31 van Gorp S, Kessels AG, Joosten EA, et al. Pain prevalence and its determinants after spinal cord injury: a systematic review. Eur J Pain 2015;19:5-14.

32 Hatem SM, Attal N, Ducreux D, et al. Clinical, functional and structural determinants of central pain in syringomyelia. Brain 2010;133:3409-22.

33 Cruz-Almeida Y, Felix ER, Martinez-Arizala A, et al. Decreased spinothalamic and dorsal column medial lemniscus-mediated function is associated with neuropathic pain after spinal cord injury. J Neurotrauma 2012;29:2706-15.

34 Widerström-Noga E, Cruz-Almeida Y, Felix ER, et al. Somatosensory phenotype is associated with thalamic metabolites and pain intensity after spinal cord injury. Pain 2015;156:166-74

35 Ozdemir RA, Perez MA. Afferent input and sensory function after human spinal cord injury. J Neurophysiol 2018;119:134-44.

36 Al-Chalabi M, Gupta S. Neuroanatomy, spinothalamic tract. Treasure Island, FL: StatPearls, 2019

37 Finnerup N, Sørensen L, Biering-Sørensen F, et al. Segmental hypersensitivity and spinothalamic function in spinal cord injury pain. Exp Neurol 2007;207:139-49.

38 Onifer SM, Smith GM, Fouad K. Plasticity after spinal cord injury: relevance to recovery and approaches to facilitate it. Neurotherapeutics 2011;8:283-93.

39 Tsuda M, Koga K, Chen T, et al. Neuronal and microglial mechanisms for neuropathic pain in the spinal dorsal horn and anterior cingulate cortex. J Neurochem 2017; 141:486-98.

40 Masuda T, Ozono Y, Mikuriya S, et al. Dorsal horn neurons release extracellular ATP in a VNUT-dependent manner that underlies neuropathic pain. Nat Commun 2016;7:12529

41 Jackson AB, Dijkers M, Devivo MJ, et al. A demographic profile of new traumatic spinal cord injuries: change and stability over 30 years. Arch Phys Med Rehabil 2004:85:1740-8

42 Finnerup NB, Gyldensted C, Nielsen E, et al. MRI in chronic spinal cord injury patients with and without central pain. Neurology 2003;61:1569-75. 